\title{
The impact of key parameters on the cycle efficiency of multi-stage RCAES system
}

\author{
Bin LIU, Laijun CHEN, Shengwei MEI ( $₫)$, \\ Feng LIU, Junjie WANG, Sixian WANG
}

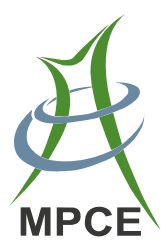

\begin{abstract}
Due to the uncertainty and anti-peaking nature, large scale integration of renewable energy imposes great challenges to the operation and dispatch of power systems. Compressed air energy storage (CAES) system provides new ideas to solve this problem as its characteristics of fast regulating, flexible location and long-service life. Especially, regenerative compressed air energy storage (RCAES) system is widely concerned as its capability of heat recovery in the compression process. The cycle efficiency is a key indicator of RCAES system which can be significantly impacted by the key parameters of the systems including compression ratio, exhaust air pressure of throttle (EAPT) and the maximum working pressure (MWP) of compressed air storage vessel (CASV). However, current research mostly focuses on the thermodynamic process and few studies have focused on the impact of key parameters on RCAES system. Based on the efficiency evaluation method which was formulated through the electricalmechanical-thermal dynamic process and measurable parameters, the impact of key parameters on the cycle efficiency of RCAES system is analyzed in this paper and a practical RCAES design scheme is adopted for case study.
\end{abstract}

Keywords RCAES, Efficiency evaluation, Parameters analysis, Energy conversion, Energy storage

CrossCheck date: 20 November 2014

Received: 27 May 2014/ Accepted: 4 December 2014/Published online: 17 December 2014

(C) The Author(s) 2014. This article is published with open access at Springerlink.com

B. LIU, L. CHEN, S. MEI, F. LIU, State Key Lab of Power

Systems, Department of Electrical Engineering, Tsinghua

University, Beijing 100084, China

$(\bowtie)$ e-mail: meishengwei@tsinghua.edu.cn

J. WANG, S. WANG, Key Laboratory of Cryogenics, Technical

Institute of Physics and Chemistry, Chinese Academy of

Sciences, Beijing 100190, China

\section{Introduction}

Integration of large-scale renewable energy has imposed great challenges on power system operation due to its uncertainty and anti-peaking nature. The load regulating capacity was seriously deficient in Gansu power grid with $5.409 \mathrm{GW}$ integration of wind power by the end of 2011 [1]. In order to effectively cope with the uncertainty and antipeaking nature of renewable energy, viable option includes the innovative schedule strategy to optimize system reserves, Energy Storage System (ESS) and so on [2-7].

Chemical battery, pumped hydro, flying wheel, superconducting and compressed air are main patterns of energy storage currently. Chemical battery is the most mature one but with high cost and environmental risk. Pumped hydro energy storage is widely applied in power systems. However, its location depends on the geographical environment. Large-scale applications of flying wheel or superconducting energy storage are very limited in power system practically [8]. Compressed air energy storage (CAES) system is widely concerned in recent years due to its characteristics of high-capacity, long-service life and flexible location of which Huntorf in Germany and McIntosh in USA are representative systems globally [9].

Most existing CAES systems are with combustion process which could improve the cycle efficiency with compressed air. However, such CAES system relies greatly on natural gas supply [10]. To overcome the above disadvantages, regenerative compressed air energy storage (RCAES) system is concerned in energy storage area both in science and engineering due to its capability of reusing compression heat in expansion process. The RCAES system can conspicuously improve the system efficiency without any greenhouse gases emission. The expected efficiency of the typical advanced adiabatic compressed air energy storage system can be regarded as a kind of RCAES 
system under construction by RWE in Germany is $70 \%$ [11-13].

The technology project "Research of Key Technology and Engineering Application of Compressed Air Storage" (simply as RCAES project) in China is started in 2012. One of the key technologies to be concerned in the project is how to reuse the heat released in the compression process with high efficiency and explore a viable solution to the prevalent waste of renewable energy in power systems.

The cycle efficiency is a key indicator of RCAES system and can be significantly impacted by the key parameters of the system. Different kind of efficiency definitions are compared in [14] and the thermodynamic effect of advanced adiabatic compressed air energy storage system is analyzed in [15]. However, neither the efficiency evaluation method nor the impact of the parameters is concerned by any of the aforementioned references.

Based on the electric-mechanic-thermal dynamic process and measurable parameters of RCAES system, we proposed an efficiency evaluation method of RCAES system in [16] previously. One advantage of the proposed method is that it can be extended to study the impact of key parameters on the cycle efficiency of RCAES system, which is to be fulfilled in this paper. The key parameters of a RCAES system in this paper includes compression ratio, exhaust air pressure of throttle (EAPT) and the maximum working pressure (MWP) of compressed air storage vessel (CASV).

The remaining of this paper is organized as follows. Firstly, the efficiency evaluation method proposed in [16] will be briefly reviewed and the analytical method will also be formulated theoretically. Then the typical design scheme of RCAES project is studied. Finally, the conclusions are drawn.

\section{Efficiency evaluation of RCAES system}

\subsection{Main assumptions}

A simplified structure of RCAES system is showed in Fig. 1 including four main parts: compression, thermal energy storage (TES), CASV and expansion. The main assumptions in the system are as follow:

1) Ideal gas during the operation;

2) The air mass flow rate in compression and expansion process is known and constant in operation;

3) The isothermal model is adopted for CASV in which the temperature is the same as ambient circumstances;

4) The temperature and pressure of compressed air after throttling become constant.

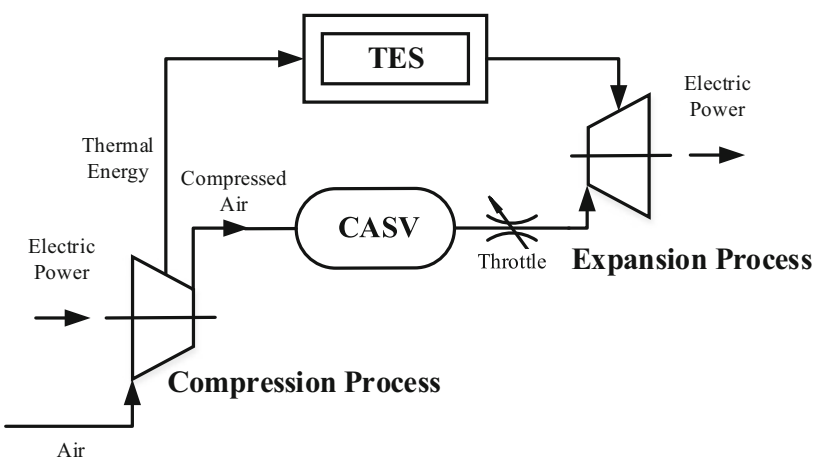

Fig. 1 Design structure of RCAES system

To facilitate the energy conversion process, without loss of generality, we further assume that:

1) The compression process starts when the pressure of CASV reaching its minimum value $p_{\text {str }}^{\min }$ and ends when the pressure reaching its maximum value $p_{\text {str }}^{\max }$. Besides, EAPT denoted by $p_{\text {eap }}$ is equal to $p_{\text {str }}^{\min }$.

2) The compressor consists of $N$ stages of which stage 1 to stage $N-1$ are steady stages and the last stage, i.e. stage $N$ is an unsteady stage. The exhaust air pressure of stage $N-1$ denoted by $p_{\text {cou }, \mathrm{N}-1}$, is lower than the MWP of CASV, i.e. $p_{\mathrm{str}}^{\min }<p_{\mathrm{cou}, \mathrm{N}-1}<p_{\mathrm{str}}^{\max }$, while the exhaust air pressure of the last stage denoted by $p_{\text {cou, } N}$ is larger than the MWP of CASV, i.e. $p_{\mathrm{cou}, \mathrm{N}}>p_{\mathrm{str}}^{\max }$.

Hence, all the stages of compressor will work simultaneously and the previous $N-1$ stages are steady while the last stage is unsteady with time-variant pressure with the same value as CASV. Once the above assumptions are not satisfied for a practical RCAES system, the compression process can be formulated in a multi-period way with the same methodology. Furthermore, TES can be controlled to keep the inlet air temperature of all compression stages the same value except the $1^{\text {st }}$ one in practical engineering and the corresponding temperature is denoted by $T_{\text {cin }}$ in this paper.

\subsection{Efficiency formulation of the whole RCAES system}

Traditional and general efficiency evaluation method for CAES system is indicated by the ratio of the output electric energy and input electric energy. However, this method is merely applicable to systems already in operation and can hardly be extended to analyze or optimize the key parameters of the system. To overcome the deficiency, we proposed an efficiency evaluation method based on the energy conversion of compression and expansion process in [16]. 


\subsubsection{Energy conversion in compression process}

In compression process, the RCAES system is charged with electric power of which one part is stored in highpressure air as molecular potential energy and the other part is stored in high-temperature water as thermal energy. However, the close relationship between electric power and compression work can be illustrated with the compression efficiency $\eta_{\text {com }}$. The compression efficiency is defined as the ratio of compression work and electric power charged in the compression process and is regarded as a known parameter when the system efficiency is analyzed in this paper.

As the isothermal model is adopted for CASV, the operation time (denoted as $t_{\text {com }}$ with the unit of $s$ ) of the compression process can be illustrated as a function of $p_{\text {str }}$ based on the state equation of ideal gas under normal circumstances which is expressed as follows:

$t_{\mathrm{com}}=\frac{V_{\mathrm{str}}\left(p_{\mathrm{str}}-p_{\mathrm{str}}^{\mathrm{min}}\right)}{q_{\mathrm{com}} R_{\mathrm{g}} T_{\mathrm{str}}}$

where $p_{\text {str }}, V_{\text {str }}, T_{\text {str }}$ are pressure, volume and temperature of CASV with units of $\mathrm{Pa}, \mathrm{m}^{3}, \mathrm{~K}$ separately; $q_{\text {com }}$ is the exhaust air rate of compressor with unit of $\mathrm{kg} / \mathrm{s} ; R_{\mathrm{g}}=$ $287.1 \mathrm{~J} /(\mathrm{kg} \cdot \mathrm{K})$ is a gas constant of air under normal circumstances [17].

When the $p_{\text {str }}$ reaches the MWP of CASV, the value of $t_{\text {com }}$ equals the operation time of compression process.

In the compression process, the compression work of steady stages and unsteady stage should be calculated separately as the compression power of unsteady stage changes dynamicly in accordance with the pressure of CASV.

The total compression power (denoted by $P_{\text {std }}$ with the unit of $\mathrm{kW}$ ) of steady stages $1 \sim N-1$ remain unchanged and can be expressed as follows [18]:

$P_{\text {std }}=\frac{q_{\mathrm{com}} R_{\mathrm{g}} \gamma}{10^{3} \times(\gamma-1)}\left(\lambda^{\frac{\gamma-1}{\gamma}}-1\right)\left[T_{\mathrm{c} 1}+(N-2) T_{\mathrm{cin}}\right]$

where $\lambda$ is the ratio of each stage of compressor satisfying $\lambda>1 ; T_{\mathrm{c} 1}$ is the inlet temperature of the $1^{\text {st }}$ stage and $\gamma$ is the polytropicexponent of each stage.

The total compression work (denoted by $W_{\text {std }}$ with the unit of $\mathrm{kWh}$ ) of steady stages can then be illustrated as follows after the multiplication of (1) and (2):

$$
\begin{aligned}
W_{\text {std }}\left(p_{\text {str }}\right) & =P_{\text {std }} t_{\text {com }} / 3600 \\
& =\frac{V_{\text {str }} \gamma\left(p_{\text {str }}-p_{\text {str }}^{\min }\right)\left(\lambda^{\frac{\gamma-1}{\gamma}}-1\right)}{3.6 \times 10^{5} T_{\mathrm{str}}(\gamma-1)}\left[T_{\mathrm{c} 1}+(N-2) T_{\text {cin }}\right]
\end{aligned}
$$

Similarly, the last stage of compressor is unsteady of which the exhaust air pressure is the same with CASV. The compression power (denoted by $P_{\mathrm{utd}}\left(p_{\mathrm{str}}\right)$ with the unit of kW) can be expressed as [18]:

$P_{\text {utd }}\left(p_{\text {str }}\right)=\frac{q_{\text {com }} R_{\mathrm{g}} T_{\text {cin }} \gamma}{10^{3}(\gamma-1)}\left[\left(\frac{p_{\text {str }}}{p_{\text {cin }, \mathrm{N}}}\right)^{\frac{\gamma-1}{\gamma}}-1\right]$

where $p_{\text {cin,N }}$ is the inlet pressure of stage $N$.

Then, the compression work (denoted by $W_{\text {utd }}\left(p_{\text {str }}\right)$ with the unit of $\mathrm{kWh}$ ) can be obtained after integrating the compression power on time basis.

$$
\begin{aligned}
W_{\text {utd }}\left(p_{\text {str }}\right) & =\int \frac{P_{\mathrm{utd}}}{3600} \mathrm{~d} t=\int \frac{V_{\text {str }} P_{\mathrm{utd}} / 3600}{q_{\mathrm{com}} T_{\mathrm{str}} R_{\mathrm{g}}} \mathrm{d} p \\
& =\alpha_{1}\left[\left(\frac{p_{\mathrm{str}}}{p_{\mathrm{cin}, \mathrm{N}}}\right)^{\frac{2 \gamma-1}{\gamma}}-\left(\frac{p_{\mathrm{str}}^{\min }}{p_{\mathrm{cin}, \mathrm{N}}}\right)^{\frac{2 \gamma-1}{\gamma}}\right]-\alpha_{2}\left(p_{\mathrm{str}}-p_{\mathrm{str}}^{\min }\right)
\end{aligned}
$$

where $\alpha_{1}=\frac{T_{\text {cin }} V_{\text {str }} \gamma^{2} \lambda^{N-1} p_{\text {cin }, 1}}{3.6 \times 10^{-5} T_{\text {str }}(2 \gamma-1)(\gamma-1)}, \quad \alpha_{2}=\frac{T_{\text {cin }} V_{\text {str }} \gamma}{3.6 \times 10^{-5} T_{\text {str }}(\gamma-1)}$.

In summary, the compression power and work can be expressed as a function of $p_{\text {stor }}$ :

$P_{\text {com }}\left(p_{\text {str }}\right)=P_{\text {std }}+P_{\text {utd }}\left(p_{\text {str }}\right)$

$W_{\text {com }}\left(p_{\text {str }}\right)=W_{\text {std }}\left(p_{\text {str }}\right)+W_{\text {utd }}\left(p_{\text {str }}\right)$

In (6), $P_{\text {std }}$ denotes the total compression power of steady stages which is independent of $p_{\text {str }}$ and $P_{\mathrm{utd}}\left(p_{\text {str }}\right)$ denotes the compression power of unsteady stage changing along with $p_{\text {str }}$. The total compression power $P_{\text {com }}\left(p_{\text {str }}\right)$ in the compression process is a summation of compression powers of steady and unsteady stages and thus a function of $p_{\text {str }}$. The total compression work $W_{\text {com }}\left(p_{\text {str }}\right)$ can be explained similarly.

Then, the electric energy (denoted by $E_{\mathrm{com}}^{\max }$ with the unit of $\mathrm{kWh}$ ) charged during the compression process can be expressed as:

$E_{\mathrm{com}}^{\mathrm{max}}=\frac{W_{\mathrm{com}}\left(p_{\mathrm{str}}^{\max }\right)}{\eta_{\mathrm{com}}}$

\subsubsection{Energy conversion in expansion process}

In the expansion process, the high-pressure air changes into constant-temperature and isopiestic air after throttling, and then drives the turbine to generate electric power after exchanging heat with TES system. Similar to the compression process, the relationship of expansion work and electric energy can be illustrated by the expansion efficiency $\eta_{\text {tur }}$ which is defined as the ration of discharged electric power and the expansion work in this paper. The expansion efficiency is also regarded as a known parameter when analyzing the system efficiency in this paper.

Due to the throttling effect, all stages are steady in expansion process. Supposing the tempreture of inlet air and polytropicexponent are indicated by $T_{\text {tin }}$ and $\mu$ 
separately, the total expansion power (denoted by $P_{\text {tur }}$ with the unit of $\mathrm{kW}$ ) can be expressed as follows [18]:

$P_{\mathrm{tur}}=-\frac{M q_{\mathrm{tur}} R_{\mathrm{g}} T_{\mathrm{tin} \gamma}}{10^{3}(\mu-1)}\left(\phi^{\frac{\mu-1}{\mu}}-1\right)$

where $M$ is the total number of stage in the turbine; $\phi$ is the expansion ratio of each stage satisfying $\phi<1 ; q_{t u r}$ is the exhaust air rate of turbine with the unit of $\mathrm{kg} / \mathrm{s}$.

The expansion time (denoted as $t_{\text {tur }}$ with the unit of $s$ ) can be obtained in the same way as described in 2.2.1 showed as follows:

$t_{\text {tur }}=\frac{\left(p_{\mathrm{str}}^{\max }-p_{\mathrm{str}}\right) V_{\mathrm{str}}}{q_{\mathrm{tur}} R_{\mathrm{g}} T_{\mathrm{str}}}$

Hence, the expansion work (denoted by $W_{\text {tur }}$ with the unit of $\mathrm{kWh}$ ) can be expressed as a function of $p_{\text {str }}$ and showed as follows:

$W_{\text {tur }}\left(p_{\text {str }}\right)=P_{\text {tur }} t_{\text {tur }} / 3600$

$=\frac{M T_{\text {tin }} \mu V_{\text {str }}\left(p_{\mathrm{str}}^{\max }-p_{\text {str }}\right)}{3.6 \times 10^{5} T_{\mathrm{str}}(\mu-1)}\left(1-\phi^{\frac{\mu-1}{\mu}}\right)$

The electric energy (denoted by $E_{\text {tur }}^{\max }$ with the unit of $\mathrm{kWh}$ ) discharged by the RCAES system can then be expressed as the multiplication of expansion work and expansion efficiency:

$E_{\text {tur }}^{\max }=W_{\text {tur }}\left(p_{\text {str }}\right) \eta_{\text {tur }}$

Finally, the efficiency of RCAES system can be calculated as (12) and the details can be found in Appendix A.

$\eta_{\mathrm{RCAES}}=\frac{E_{\mathrm{tur}}^{\max }}{E_{\mathrm{com}}^{\max }}=\frac{W_{\mathrm{tur}}\left(p_{\mathrm{str}}^{\min }\right)}{W_{\mathrm{com}}\left(p_{\mathrm{str}}^{\max }\right)} \eta_{\mathrm{com}} \eta_{\mathrm{tur}}$

According to the above formulas, the efficiency of the RCAES system is a nonlinear function of $\lambda, p_{\text {eap }}$ and $p_{\text {str }}^{\max }$.

2.3 The impact of key parameters on the efficiency of RCAES systems

\subsubsection{The impact of compression ratio $\lambda$}

When the compression ratio increases, more electric energy will be charged into the RCAES system and more thermal energy will be released by the compressor and absorbed by the TES system in compression process. Consequently, more thermal energy will be reused by the turbine and more electric energy will be discharged in the expansion process.

The relationship between the inlet air temperature of compressor $T_{\text {cin }}$ and the exhaust air temperature in steady stage, denoted by $T_{\text {cou }}$, can be showed as follows:
$T_{\text {cou }}=T_{\operatorname{cin}} \lambda^{\frac{\gamma-1}{\gamma}}$

To establish the relationship of $T_{\text {cou }}$ and the inlet air temperature of the turbine $T_{\text {tin }}$, a reasonable and applicable technique in engineering is assuming the gap being a constant, indicated by $\Delta T$, which represents the efficacy of the TES. The smaller the value of $\Delta T$ is, the better the efficacy of the TES is. Therefore, the relationship of $T_{\text {cou }}$ and $T_{\text {tin }}$ can be expressed as follows:

$T_{\text {tin }}=T_{\text {cou }} \lambda^{\gamma-1} \gamma+\Delta T$

By substituting (14) into (12), we can get the mathematical expression of the efficiency of RCAES system (see Appendix).

Additionally, to ensure all of the compressor working properly, the exhaust pressure of the stage $N-1$ should be no more than the MWP of CASV showed as follows:

$p_{\text {cin }, 1} \lambda^{N-1}<p_{\text {str }}^{\max }$

Otherwise the last stage, i.e. stage $N$ will be useless. Similarly, stage $N$ of the compressor should be larger than the MWP of CASV showed as follows implying that the maximum pressure of CASV can only be reached when all stages work simultaneously.

$p_{\text {cin }, 1} \lambda^{N}>p_{\text {str }}^{\max }$

Therefore, the compression ratio $\lambda$ should satisfy

$\sqrt[N]{\frac{p_{\mathrm{str}}^{\max }}{p_{\mathrm{cin}, 1}}}<\lambda<\sqrt[N-1]{\frac{p_{\mathrm{str}}^{\max }}{p_{\mathrm{cin}, 1}}}$

\subsubsection{The impact of EAPT $p_{\text {eap }}$}

On one hand, the variety of EAPT will affect the operation time of both compression and expansion process according to (1) where $p_{\mathrm{str}}^{\min }=p_{\text {eap }}$ under given assumptions. On the other hand, as the exhaust air pressure of the turbine's last stage $p_{\text {tou }, M}$ and total stage number of the turbine $M$ are both fixed, the relationship of the expansion ratio $\phi$ can be expressed as

$\phi=\sqrt[M]{\frac{p_{\text {tou }, \mathrm{M}}}{p_{\text {eap }}}}$

In the above formulation, the reason that $p_{\text {tou, }}$ can be treated as a constant is that the exhaust air pressure of the last stage of turbine and the air pressure of ambient circumstances are approximately equal in the practical engineering applications.

By substituting (16) into (12), we can get the expression of the efficiency of RCAES system over $p_{\text {eap }}$ (see Appendix). 


\subsubsection{The impact of the MWP of CASV $p_{\mathrm{str}}^{\max }$}

Similar to the impact of EAPT, the MWP of CASV will affect the efficiency of RCAES system indirectly. As the system efficiency is a nonlinear function of $p_{\mathrm{str}}^{\max }$, the specific impact will be analyzed in case study.

Additionally, $p_{\text {str }}^{\max }$ also should satisfy the following constraint to guarantee the efficacy of every stage of the compressor, i.e., the MWP of CASV can be reached only when all stages work simultaneously.

$p_{\text {cin }, 1} \lambda^{N-1}<p_{\text {str }}^{\max }<p_{\text {cin }, 1} \lambda^{N}$

\section{Case study}

\subsection{System introduction}

The typical design scheme of the RCAES project showed in Fig. 2. According to Fig. 2, the compressor consists of 5 stages and the turbine consists of 3 stages. The basic parameters are showed in Table 1 under the comprehensive consideration of the operation performance and construction cost of the RCAES system.

\subsection{Efficiency of RCAES system with basic parameters}

According to (1), the compression process can be divided into two periods. The first one takes $3.65 \mathrm{~h}$ and the
Table 1 Parameters of practical RCAES system

\begin{tabular}{llll}
\hline Parameter & Value & Parameter & Value \\
\hline$T_{\text {c1 }}$ & $25{ }^{\circ} \mathrm{C}$ & $p_{\text {str }}^{\min }\left(p_{\text {eap }}\right)$ & $2.5 \mathrm{MPa}$ \\
$p_{\text {cin }, 1}$ & $0.1 \mathrm{MPa}$ & $p_{\text {str }}^{\max }$ & $10 \mathrm{MPa}$ \\
$\eta_{\text {com }}$ & $75 \%$ & $\lambda$ & 3 \\
$\eta_{\text {tur }}$ & $75 \%$ & $\phi$ & $1 / 3$ \\
$T_{\text {str }}$ & $20{ }^{\circ} \mathrm{C}$ & $q_{\text {com }}$ & $0.46 \mathrm{~kg} / \mathrm{s}$ \\
$\Delta T$ & $40{ }^{\circ} \mathrm{C}$ & $q_{\text {tur }}$ & $2.41 \mathrm{~kg} / \mathrm{s}$ \\
$\gamma$ & 1.4 & $\mu$ & 1.4 \\
$p_{\text {tou }, \mathrm{M}}$ & $0.11 \mathrm{MPa}$ & $V_{\text {str }}$ & $100 \mathrm{~m}^{3}$ \\
$T_{\text {cin }}$ & $86.35{ }^{\circ} \mathrm{C}$ & & \\
\hline
\end{tabular}

second one takes $1.36 \mathrm{~h}$. The operation time of the expansion process is $1.14 \mathrm{~h}$ according to (9). Fig. 3 and Fig. 4 show the compression power/energy process changing with $p_{\text {str }}$ in the compression process.

As showed in Fig. 3 and Fig. 4, the working pressure of CASV $p_{\text {str }}$ increases over compression power $P_{\text {com }}$ and compression work $W_{\text {com }}$ nonlinearly. In the compression process, the maximum electric power and electric energy charged into the RCAES system is $283.90 \mathrm{~kW}$ and $1407.55 \mathrm{~kW}$ separately. The electric power discharged by the RCAES system remains $498.17 \mathrm{kWh}$ and the maximum electric energy discharged is $567.92 \mathrm{~kW}$. According to (12), the efficiency of RCAES system is $40.35 \%$ in the given case.

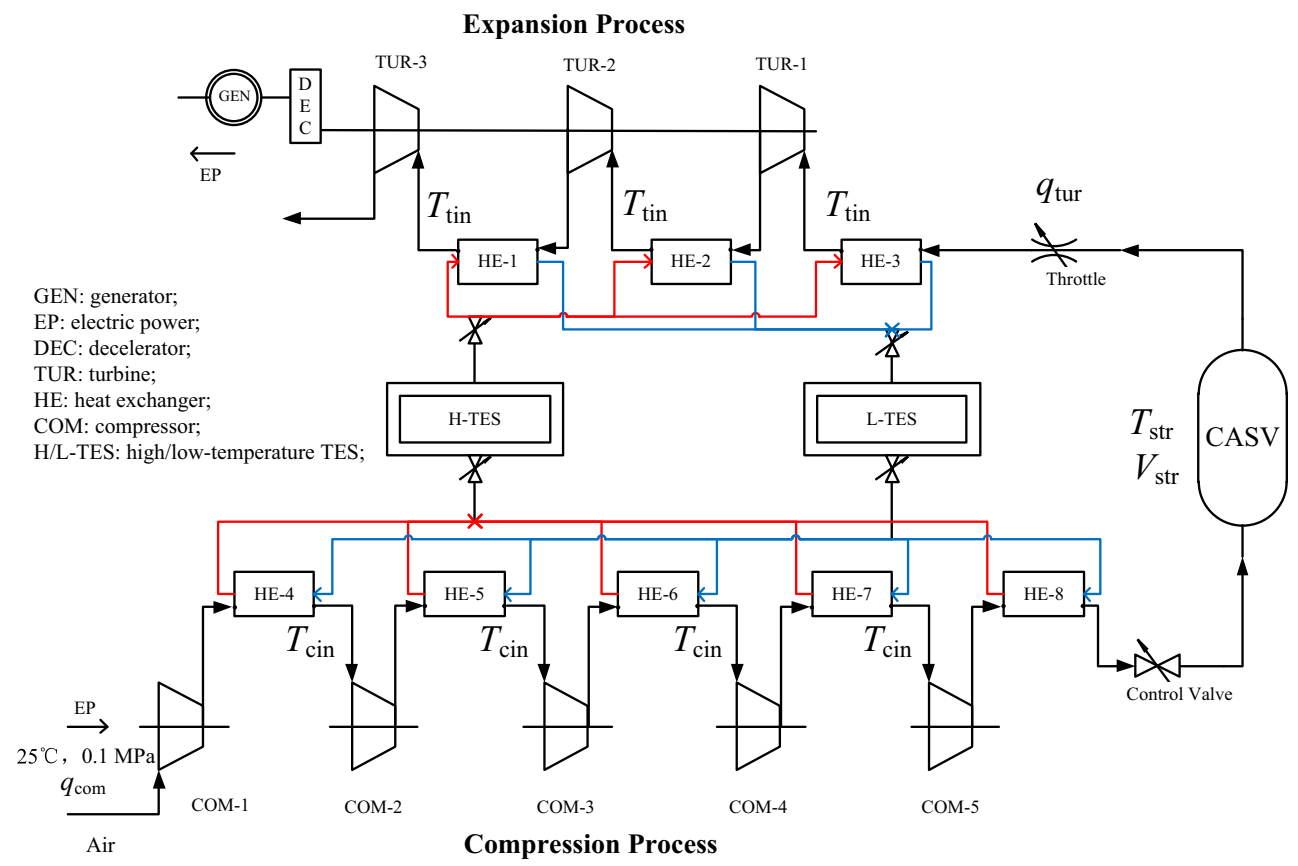

Fig. 2 Design scheme of RCAES project 


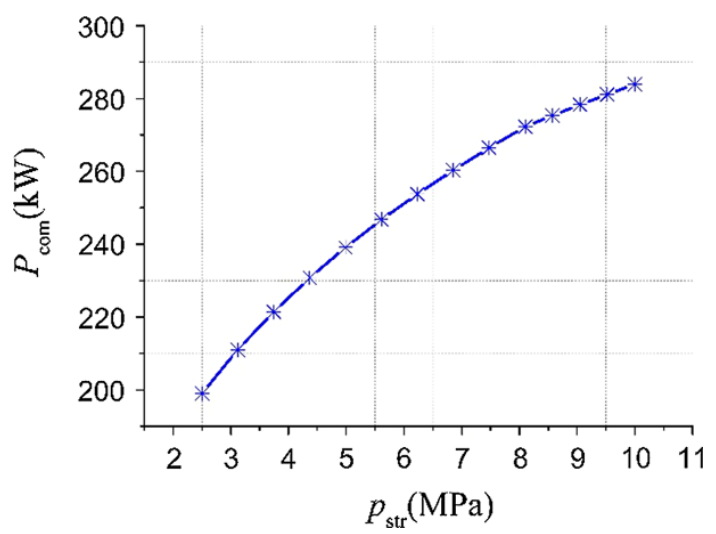

Fig. 3 Compression power $\boldsymbol{P}_{\text {com }}$ with different $\boldsymbol{p}_{\text {str }}$ in compression process

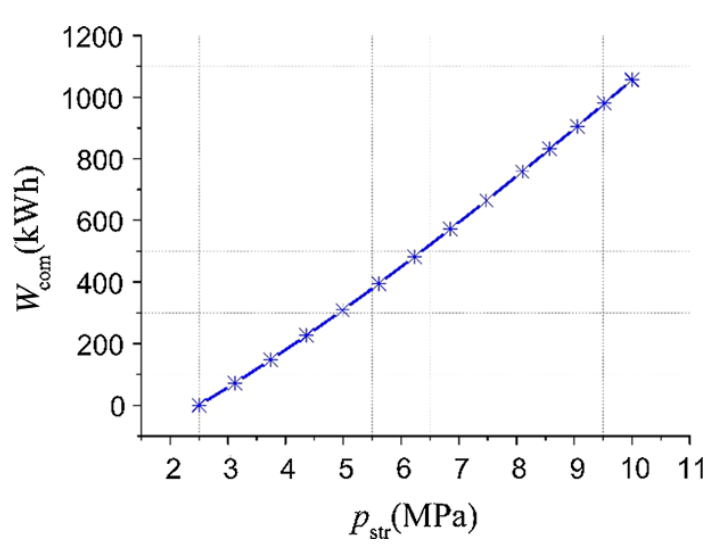

Fig. 4 Compression work $\boldsymbol{W}_{\text {com }}$ with different $\boldsymbol{p}_{\text {str }}$ in compression process

\subsection{The impact of compression ratio}

In the formulation of this part, all parameters excluding the compression ratio $\lambda$ of the RCAES system are given as basic parameters given in Table 1 and $\lambda$ must satisfy the following condition according to (15).

$2.5119 \approx \sqrt[N]{\frac{p_{\mathrm{str}}^{\max }}{p_{\mathrm{cin}, 1}}}<\lambda<\sqrt[N-1]{\frac{p_{\mathrm{str}}^{\max }}{p_{\mathrm{cin}, 1}}} \approx 3.1623$

Thus, $\lambda$ will be fixed as the following given values successively:

$\lambda=2.6,2.7,2.8,2.9,3.0,3.1$

The electric energy charged in compression process and discharged in expansion process with different $\lambda$ are given in Fig. 5 and Fig. 6 and both of them are monotonically increasing with $\lambda$. The efficiency of RCAES system, i.e., the ratio of $E_{\mathrm{tur}}^{\max }$ and $E_{\mathrm{com}}^{\max }$ is given in Fig. 7.

As showed in Fig. 7, the RCAES system's efficiency increases approximately linearly over $\lambda$. This is partly

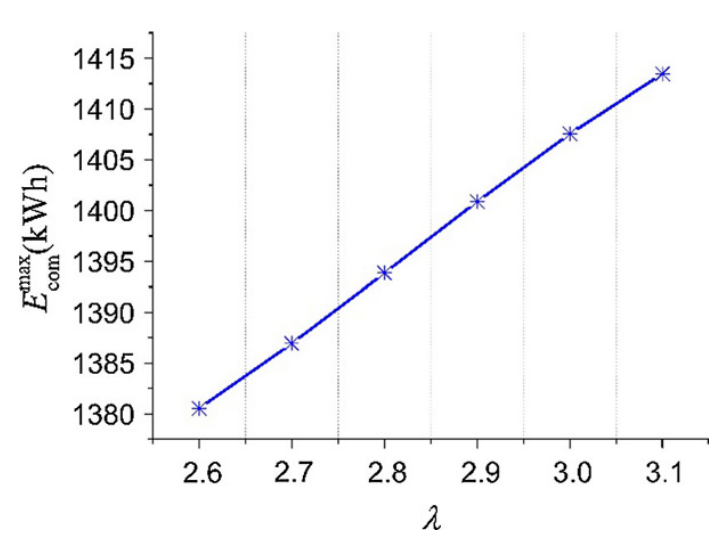

Fig. 5 Electric energy exchanged with different $\lambda$ in compression processes

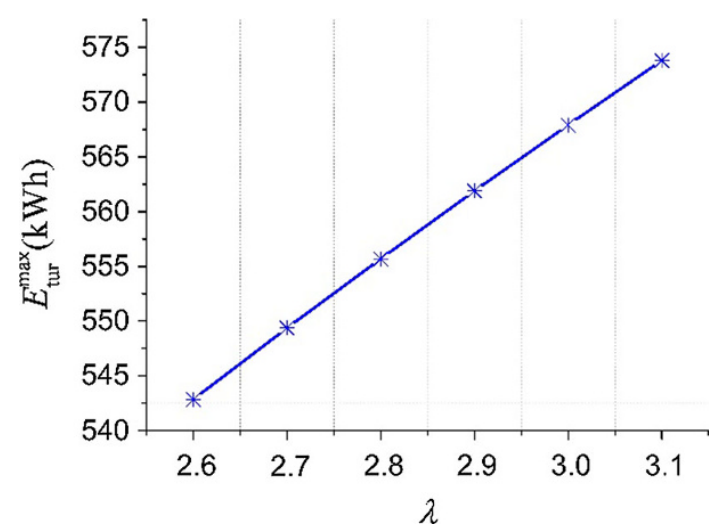

Fig. 6 Electric energy exchanged with different $\lambda$ in expansion processes

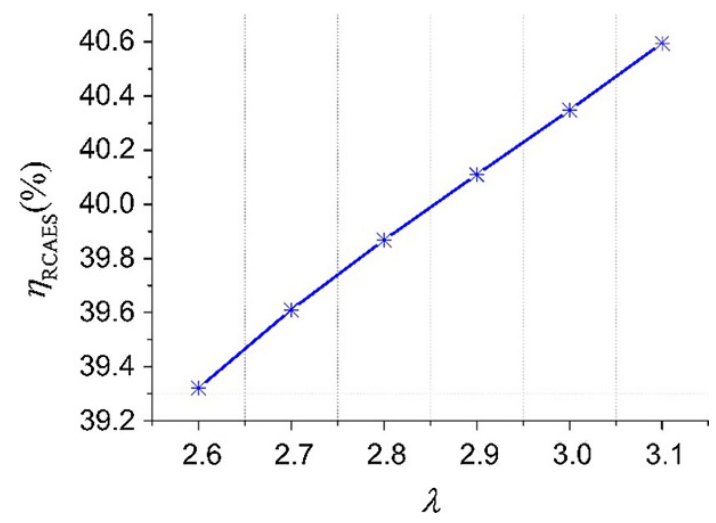

Fig. 7 Efficiency of RCAES system with different $\lambda$

because of the narrow feasible region of $\lambda$ under the given design scheme, although the nonlinearity is indicated by the formulas in Appendix.

As the compression ratio $\lambda$ increases, more thermal energy is released, resultantly, raising the temperature of H-TES. Accordingly, the rising inlet air temperature of the turbine implies that more electric energy will be discharged in the expansion process. The simulation result shows that 
the incremental electric energy discharged in expansion process is larger than the incremental electric energy charged in compression process as $\lambda$ increases, i.e., the efficiency of the RCAES system is increased.

\subsection{The impact of EAPT}

Although $p_{\text {eap }}$ can be arbitrary value in the range of $0.1 \mathrm{MPa} \sim 10 \mathrm{Mpa}$ theoretically, $p_{\text {eap }}$ will be fixed as the following given values successively in our formulation:

$p_{\text {eap }}=2.5 \mathrm{Mpa}, 3 \mathrm{Mpa}, 3.5 \mathrm{Mpa}, \cdots, 6.5 \mathrm{Mpa}, 7 \mathrm{Mpa}$

The simulation result is showed in Fig. 8 and Fig. 9, in which $E_{\mathrm{com}}^{\max }$ and $E_{\mathrm{tur}}^{\max }$ decrease over $p_{\text {eap }}$ linearly and nonlinearly separately. As the change rate of $E_{\text {tur }}^{\max }$ is smaller than $E_{\mathrm{com}}^{\max }$, the efficiency of the system increases nonlinearly and there is less energy loss with bigger value of $p_{\text {eap }}$, which is consistent with the simulation result in Fig. 10.

The energy charged into RCAES system is stored in TES as high-temperature water and in CASV as highpressure air. Given the volume of the CASV system, more

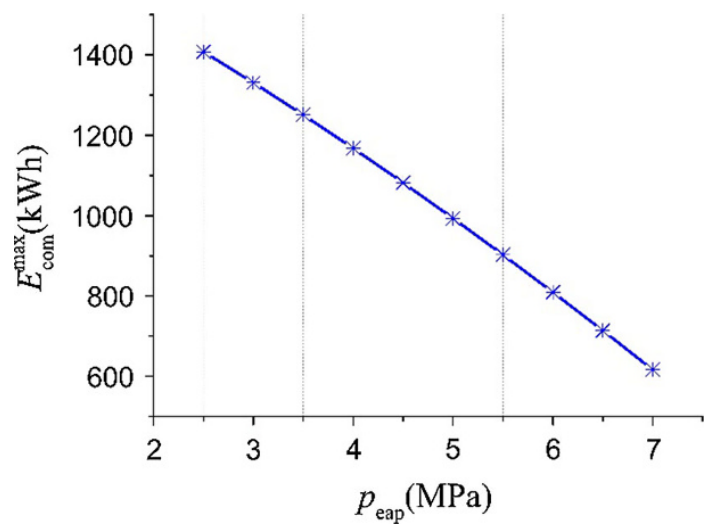

Fig. 8 Electric energy exchanged with different $\boldsymbol{p}_{\text {eap }}$ in the compression process

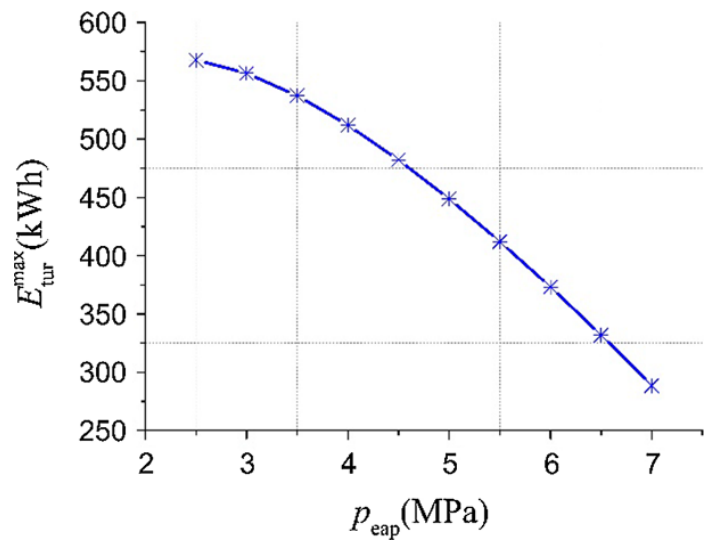

Fig. 9 Electric energy exchanged with different $\boldsymbol{p}_{\text {eap }}$ in the expansion process energy will be stored with higher pressure. However, the high-pressure air becomes isothermal and isopiestic after throttling resulting in the decrement of compressed air's capability of doing work. Furthermore, more energy loss will be caused with larger difference of the pressure.

\subsection{The impact of the MWP of CASV}

According to (17), $p_{\mathrm{str}}^{\max }$ must satisfy the following condition:

$8.1 \mathrm{MPa}=p_{\text {cin }, 1} \lambda^{N-1}<p_{\text {str }}^{\max }<p_{\text {cin }, 1} \lambda^{N}=24.3 \mathrm{MPa}$

Thus, $p_{\text {str }}^{\max }$ will be fixed as the following given values successively:

$p_{\text {str }}^{\max }=10 \mathrm{MPa}, 12 \mathrm{MPa}, \cdots, 22 \mathrm{MPa}, 24 \mathrm{MPa}$

Fig. 11 and Fig. 12 show that the electric energy charged in compression process and discharged in expansion process increases approximately linearly over $p_{\mathrm{str}}^{\max }$. This results in nonlinear decrease of the efficiency of the system which is showed in Fig. 13.

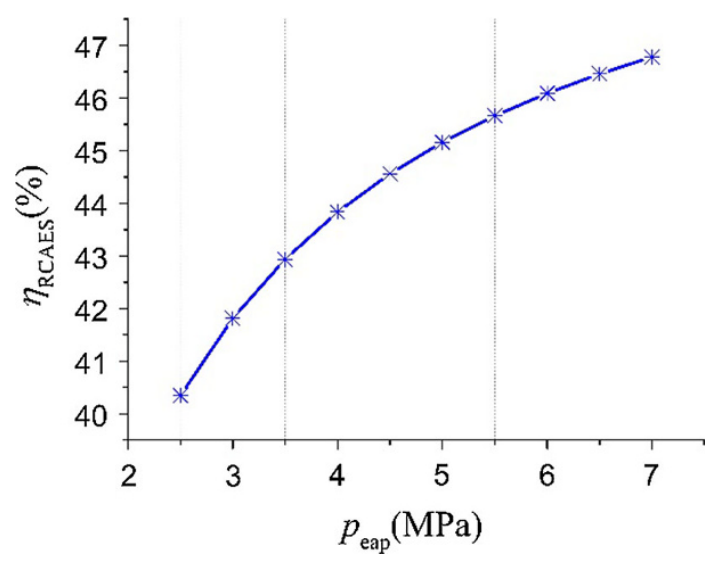

Fig. 10 Efficiency of RCAES system with different $\boldsymbol{p}_{\text {eap }}$

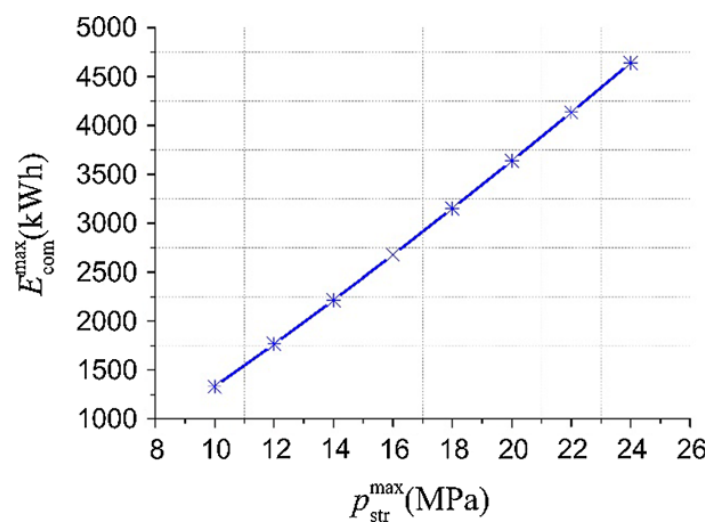

Fig. 11 Electric energy exchanged with different $p_{\text {str }}^{\max }$ in the compression process 


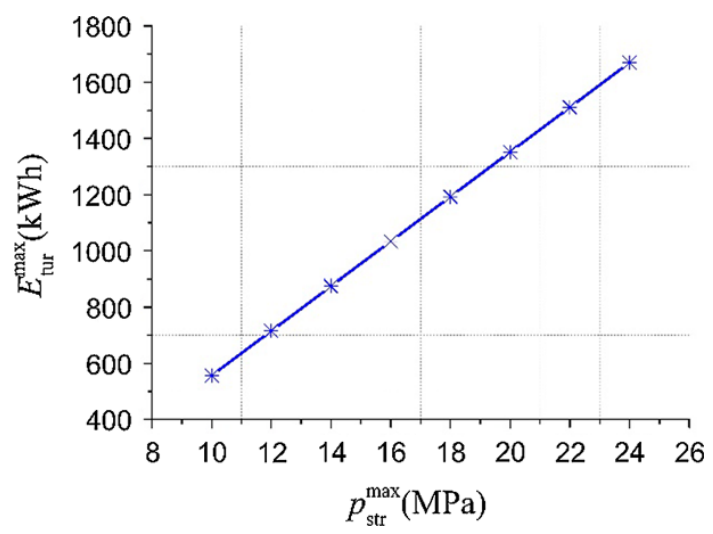

Fig. 12 Electric energy exchanged with different $\boldsymbol{p}_{\text {str }}^{\max }$ in the expansion process

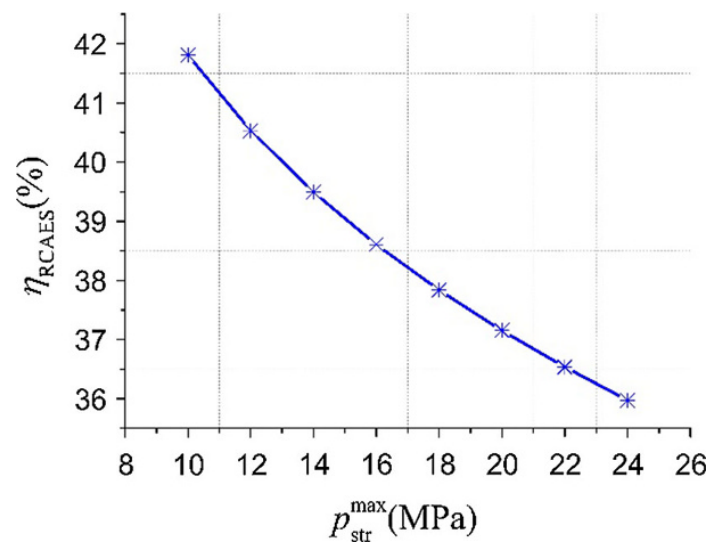

Fig. 13 Efficiency of RCAES system with different $\boldsymbol{p}_{\text {str }}^{\max }$

If $p_{\text {eap }}$ is fixed, the compressed air's capability of doing work will decrease after throttling which results in lower efficiency of the RCAES system. The simulation result also indicates that the loss is larger when MWP of the CASV is higher.

According to all of the above simulation results, higher compression ratio, higher EAPT and lower MWP of CASV in certain range are helpful to improve the cycle efficiency of the RCAES system.

\section{Conclusions}

The RCAES system is widely concerned in recent years. Based on the efficiency evaluation method, the impacts of key parameters including compression ratio, EAPT and the MWP of CASV on the cycle efficiency of RCAES system are studied in this paper. The simulation results reveal the variation of the system efficiency over these parameters. This paper provides useful information to design the
RCAES system. In addition, other key indicators of the RCAES system, e.g. maximum electric power, operation hour, maximum capacity should also be considered in practical engineering applications. The efficiency evaluation model proposed in this paper can also be used to study these influencing factors.

Acknowledgments This work is supported by National Science Foundation of China (51321005), Technology Projects of State Grid Corporation of China (SGCC) (KJ-2012-627).

Open Access This article is distributed under the terms of the Creative Commons Attribution License which permits any use, distribution, and reproduction in any medium, provided the original author(s) and the source are credited.

\section{Appendix}

According to part 2.2, the efficiency of RCAES system can be obtained by subsisting (3), (5) and (10) into (12):

$\eta_{\mathrm{RCAES}}=\frac{c_{1} z_{1}}{c_{2} z_{2}+c_{3} z_{3}-c_{4} z_{1}}$

where

$c_{1}=\frac{M T_{\text {tin }} \mu}{(\mu-1)}\left(1-\phi^{\frac{\mu-1}{\mu}}\right) ; c_{2}=\frac{\gamma\left[T_{\mathrm{c} 1}+(N-2) T_{\mathrm{cin}}\right]}{(\gamma-1)}$

$c_{3}=\frac{T_{\operatorname{cin}} \gamma^{2} p_{\operatorname{cin}, 1}}{(2 \gamma-1)(\gamma-1)} ; c_{4}=\frac{T_{\operatorname{cin} \gamma}}{(\gamma-1)}$

$z_{1}=p_{\mathrm{str}}^{\max }-p_{\mathrm{str}}^{\min } ; z_{2}=\left(\lambda^{\frac{\gamma-1}{\gamma}}-1\right)\left(p_{\mathrm{str}}^{\max }-p_{\mathrm{str}}^{\min }\right)$

$z_{3}=\lambda^{N-1}\left[\left(\frac{p_{\mathrm{str}}^{\max }}{\lambda^{N-1} p_{\mathrm{cin}, 1}}\right)^{\frac{2 \gamma-1}{\gamma}}-\left(\frac{p_{\mathrm{str}}^{\min }}{\lambda^{N-2} p_{\mathrm{cin}, 1}}\right)^{\frac{2 \gamma-1}{\gamma}}\right]$

According to part 2.3.1, the efficiency of the RCAES system over $\lambda$ can be obtained by replacing $T_{\text {tin }}$ in $c_{1}$ with (14):

$\eta_{\mathrm{RCAES}}=\frac{c_{1}^{\prime} z_{1}}{c_{2} z_{2}+c_{3} z_{3}-c_{4} z_{1}}$

where $c_{1}^{\prime}=\frac{M\left(T_{\operatorname{cin}} \lambda^{\frac{\gamma-1}{\gamma}}+\Delta T\right) \mu}{(\mu-1)}\left(1-\phi^{\frac{\mu-1}{\mu}}\right), c_{2} \sim c_{4}$ and $z_{1} \sim z_{3}$ remain unchanged.

According to part 2.3.2, replace $\phi$ in $c_{1}$ with (16), the efficiency of the RCAES system over $p_{\text {eap }}$ can be obtained as follows:

$\eta_{\mathrm{RCAES}}=\frac{c_{1}^{\prime \prime} z_{1}}{c_{2} z_{2}+c_{3} z_{3}-c_{4} z_{1}}$

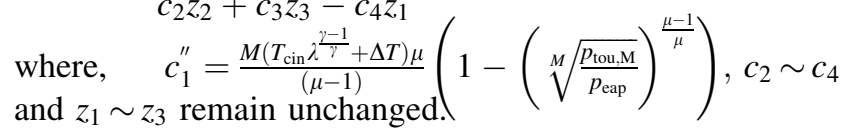




\section{References}

[1] Wang NB (2012) Transmission and consumption of large-scale wind power. China Electric Power Press, Beijing (in Chinese)

[2] Wang QF, Guan YP, Wang JH (2012) A chance-constrained two-stage stochastic program for unit commitment with uncertain wind power output. IEEE Trans Power Syst 27(1):206-215

[3] Bertsimas D, Litvinov E, Sun XA et al (2013) Adaptive robust optimization for the security constrained unit commitment problem. IEEE Trans Power Syst 28(1):52-63

[4] Jiang XD, Xie YG (2011) Impact of energy storage system on the unit commitment problem with volatile wind power. Autom Electr Power Syst 35(5):19-24 (in Chinese)

[5] Chakraborty S, Senjyu T, Yona A et al (2009) Generation scheduling of thermal units integrated with wind-battery system using a fuzzy modified differential evolution approach. In: Proceedings of the 15th international conference on intelligent system applications to power systems (ISAP'09), Curitiba, 8-12 Nov 2009, 6 pp

[6] Liu X, Xu Z, Wong KP (2013) Recent advancement on technical requirements for grid integration of wind power. J Mod Power Syst Clean Energy 1(3):216-222

[7] Xu G, Xu L, Yao L (2013) Wind turbines output power smoothing using embedded energy storage systems. J Mod Power Syst Clean Energy 1(1):49-57

[8] Zhang WL, Qiu M, Lai XK (2008) Application of energy storage technologies in power grids. Power Syst Technol 32(7):1-9 (in Chinese)

[9] Zhang XJ (2011) Investigation on compressed air storage energy storage system. PhD Thesis, Graduate University of the Chinese Academy of Sciences, Institute of Thermophysics, Beijing (in Chinese)

[10] Inage S (2009) Prospects for large-scale energy storage in decarbonised power grids. International Energy Agency, Paris

[11] Kim YM, Lee JH, Kim SJ et al (2012) Potential and evolution of compressed air energy storage-energy and exergy analysis. Entropy 14(8):1501-1521

[12] Bullough C, Gatzen C, Jakiel C, et al (2004) Advanced adiabatic compressed air energy storage for the integration of wind energy. In: Proceedings of the European wind energy conference (EWEC'04), London, 22-25 Nov 2004, 8 pp

[13] Ekman CK, Jensen SH (2010) Prospects for large scale electricity storage in Denmark. Energy Convers Manag 51(6):1140-1147

[14] Liu WY, Yang YP, Song ZP (2005) Optimization and simulation of different CAES systems. J Eng Thermophys 26(S1):25-28 (in Chinese)

[15] Kim YM, Favrat D (2010) Energy and exergy analysis of a micro-compressed air energy storage and air cycle heating and cooling system. Energy 35(1):213-220

[16] Liu B, Chen LJ, Mei SW et al (2014) An efficiency evaluation method of multi-stage RCAES system. Adv Technol Electr Eng Energy 33(8):1-6 (in Chinese)
[17] Li JP (1991) Dynamics of pneumatic transmission system. South University of Technology Press, Guangzhou (in Chinese)

[18] Shen WD, Tong JG (2007) Engineering thermodynamics, 4th edn. Higher Education Press, Beijing (in Chinese)

Bin LIU received the B.S. degree and M.S. degrees both in electrical engineering from Wuhan University, Wuhan, China and China Electric Power Research Institute, Beijing, China, in 2009 and 2012 respectively. He's now pursuing his Ph.D. degree in the Department of Electrical Engineering, Tsinghua University, Beijing, China. His main research interest includes power system optimization with renewable energy, modeling and analysis of compressed air energy storage system.

Laijun CHEN received the B.Eng. degree and Ph.D. degrees both in electrical engineering from Tsinghua University, Beijing, China, in 2006 and 2011 respectively. He is now a research assistant in the same department. His research work concerns parallel computation of electromagnetic transient in power system, modeling and analysis of compressed air energy storage system.

Shengwei MEI (SM'05) received the B.S. degree in mathematics from Xinjiang University, Urumqi, China, the M.S. degree in operations research from Tsinghua University, Beijing, China, and the Ph.D. degree in automatic control from the Chinese Academy of Sciences, Beijing, China in 1984, 1989, and 1996, respectively. He is currently a Professor of Department of Electrical Engineering at Tsinghua University. His research interests include power system analysis and control.

Feng LIU (M'12) received the B.S. and Ph.D. degrees in electrical engineering from the Department of Electrical Engineering at Tsinghua University, Beijing, China, in 1999 and 2004 respectively. $\mathrm{He}$ is currently an Associate Professor of Department of Electrical Engineering at Tsinghua University. His research concerns power system stability analysis and control.

Junjie WANG received the B.S. degree in school of mechanical engineering from Xi'an Jiaotong University, Xi'an, China, in 1983. He's now a professor in the Key Laboratory of Cryogenics, Chinese Academy of Sciences, Beijing, China. His main research interest includes energy storage system and cryogenics engineering.

Sixian WANG received the B.S. degree in school of Energy and Power Engineering from Huazhong University of Science and Technology, Wuhan, China, in 2011. He's now pursuing his Ph.D. degree in the Key Laboratory of Cryogenics, Chinese Academy of Sciences, Beijing, China. His main research interest focuses on energy storage system including compressed air energy system and liquid air energy system. 